\title{
Load Balancing in Cloud Computing
}

\author{
Sunil Tekale, J.Gladson Maria Britto, A.S. Gousia Banu
}

\begin{abstract}
Dynamic: Distributed computing is the best innovation today for every one of those individuals who needs to go with least speculation on foundation and needs to redistribute the weight of taking care of specialized issues to outsider by paying the charges for the administrations used. Today there is gigantic measure of interest from the customers to utilize cloud innovation as it gives various highlights and remove the heap of looking after foundation. This has made a tremendous measure of burden on servers. So it is must to deal with issues identified with load adjusting. This is essentially to see that the heap on a specific server is held most extreme to its edge level. So it can deal with the undertaking and furthermore can finish it in a quicker way. It limits the cost and time associated with the major computational models and improves appropriate usage of assets and framework execution. Numerous calculations are prescribed by different specialists from everywhere throughout the world to take care of the issue of burden adjusting.
\end{abstract}

In this paper, we present another calculation named as combo calculation to address the issue of burden adjusting in a cloud situation. Catchphrases - Cloud registering improvement Load Balancing Network

\section{INTRODUCTION}

Distributed computing is a recently advancing method which offers web based processing assets, stockpiling and allows clients to compose applications with upgraded adaptability, accessibility and adaptation to non-critical failure. Distributed computing is tied in with putting away the stuff on remote servers rather than on claim PCs or different gadgets. This data can be recovered utilizing the web with any gadget, wherever on the planet as long as that gadget can bolster distributed computing frameworks. The distributed computing framework is involved a front-end, which is the customer side and a back-end which is an assortment of the servers and PCs claimed by an outsider which stores the information. A focal server which is a part of the back-end pursues conventions and utilizations middleware to impart between arranged PCs. Distributed computing gathers all the registering assets and oversees them consequently Its Qualities depict a distributed computing framework: on-need self-administration, pooling of assets, access to the web, the flexibility of administration accessibility and estimation of administrations used by singular clients. Distributed computing is wherever with instruments like Google Drives supplanting Microsoft Office, Amazon Web Services supplanting conventional endeavor information stockpiling, banking sites supplanting branch workplaces and Dropbox putting away the entirety of our information and documents. The cloud even gives diverse arrangement models and administration models. The four sending models present in distributed computing are:
1. Public cloud: In people in general cloud, the cloud providerprovides assets for nothing to the general population. Any client can utilize the assets; it is unhindered. People in general cloud is associated with the open web for anybody to use.

2. Private cloud: In a private cloud, the arranging andprovisioning of the cloud are worked and claimed by the association or the outsider. Here the facilitated administrations are given to a limited number of individuals or gathering of people.

3. Community cloud: These sort of cloud infrastructuresexists for unique use by a gathering of clients. These are a gathering of clients who share a typical strategic have explicit administrative necessities, and it might be overseen by the outsider or associations.

4. Hybrid Cloud: Hybrid Cloud gives the best of aboveworlds. It is made by joining the advantage of various kinds of cloud (private cloud and open cloud). In these mists, a portion of the assets are given and oversaw by open cloud and others as a private cloud.

The three diverse help models present in distributed computing are:

1. Framework as a Service (IaaS): IaaS model providesjust the equipment and the system. It enables clients to create and introduce their working framework, programming and run any application according to their needs on cloud equipment of their own decision.

1. Platform as a Service (PaaS): In PaaS model, anoperating framework, equipment, and system are given to the client. It empowers clients to manufacture their applications on cloud utilizing provider explicit apparatuses and dialects

2. Software as a Service (SaaS): In SaaS model, a prefabricated application together with any required programming, equipment, working framework and the system is given to the client.

\section{BURDEN BALANCING}

Burden adjusting is a genuine worry in distributed computing. With the expansion in allure of distributed computing among clients, the heap on the servers and the amount of handling done is flooding definitely. There are various hubs in the cloud, and because of the arbitrary assignment of a solicitation made by the customer to any hub, the hubs become unevenly stacked.

So to dodge the condition where a few hubs are either seriously stacked or under stacked, the heap balancer will uniformly partition the remaining task at hand among every one of the hubs 3. In this way load adjusting will similarly disperse the remaining task at hand among the hubs, and it can help in limiting deferrals in correspondence, amplifying the throughput, limiting execution time and boosting asset usage 3 . 


\section{Load Balancing in Cloud Computing}

2.1 Goals of burden adjusting:

A portion of the key motivations behind a heap adjusting calculation as indicated by seem be:

1. It ought to have adaptation to internal failure.

2. It ought to be equipped for altering itself as per any change or extension in the disseminated framework arrangement 3 .

3. Regarding framework execution, it should give more noteworthy generally speaking improvement at a negligible expense.

4 .Regardless of the source of occupation it must treat all employments in the framework similarly.

4. It ought to likewise keep up framework soundness.

2.2 Issues of Load Balancing

The issues of burden adjusting are portrayed below4:

1. Load adjusting becomes basic on the grounds that, in execution, the procedures may move among hubs to guarantee equivalent outstanding task at hand on the framework 5.

2. For a heap adjusting plan to be great it ought to be adaptable, general and stable and should add insignificant overhead to the framework. These prerequisites are associated 6. 3.One of the basic parts of the planning issue is

Burden adjusting 7. The test for a planning calculation is to stay away from the contention between essentials: decency and information area.

4. Algorithms for load adjusting must be reliant on the speculation that the close by data at every hub is precise to keep away from forms from being consistently flowed the framework with no advancement 5 .

5. How to achieve an equalization in load conveyance among processors with the end goal that the calculation should be possible in the base conceivable time is one of the significant issues to determine.

6. Load adjusting and errand planning for appropriated working frameworks is an indispensable factor in net framework proficiency since $t$ he dispersed framework isn't pre-emptive and non-uniform, that is, the processors might be distinctive 7 .

2.3 Components of Load Balancing Algorithms:

A heap adjusting calculation has five significant segments 8

1. Transfer Policy: The segment of the heap adjusting calculation that picks work for moving from a nearby hub to a remote hub is expressed as Transfer arrangement or Transfer procedure.

2. Selection Policy: In this arrangement, it indicates the processors engaged with the heap trade (processor coordinating) so the general reaction time and throughput may be improved.

3. Location Policy: The bit of the heap adjusting calculation that is liable for picking a goal hub for an assignment to move is expressed as area approach or Location procedure.

4. Data Policy: The piece of the dynamic burden adjusting calculation that is responsible for social event data about the hubs present in the framework is begun to as Information Policy or Information system.

5. Burden Estimation Policy: In this approach, it decides the absolute remaining task at hand of a hub in a framework.
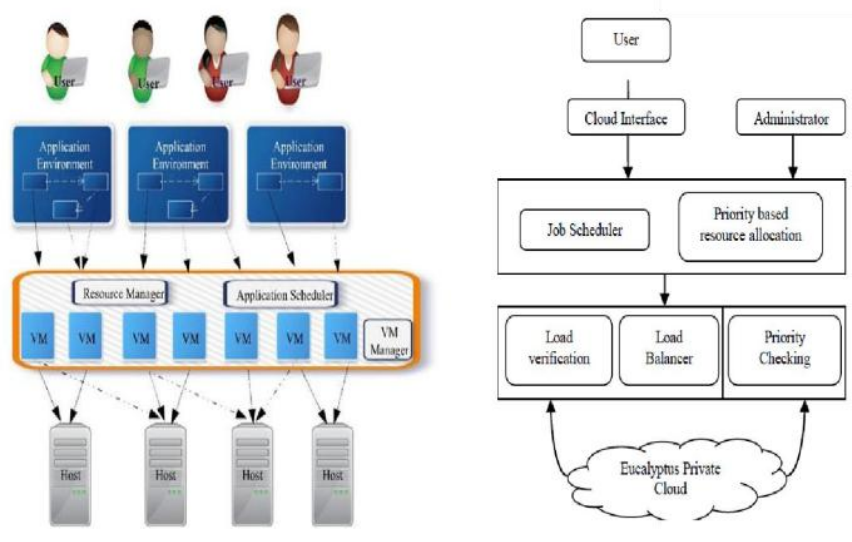

Fig: 1 shows the VM,Application,Host relationship in DC

\section{Advantages of Cloud Load Balancing}

The advantages of cloud load adjusting specifically emerge from the versatile and worldwide character of the cloud itself. The straightforwardness and speed of scaling in the cloud implies that organizations can deal with traffic spikes (like those on Cyber Monday) without corrupted execution by putting a cloud load balancer before a gathering of use cases, which can rapidly autoscale in response to the degree of interest. The capacity to have an application at various cloud center points the world over can support unwavering quality.

On the off chance that a power blackout hits the northeastern U.S. after a blizzard, for instance, the cloud load balancer can coordinate traffic away from cloud assets facilitated there to assets facilitated in different parts of the nation.

\section{Proposed Algorithm.}

The Evaluation based burden adjusting should be possible in a significantly more simpler and most straightforward way with high productivity. In this we need to simply figure the limit of every server as far as its memory, processing speed, etc. In view of the assessment of this we can give a score as far as imprints on a size of 100 . So all server farms will have server and every server will have a score dependent on the assessment of its ability.

The concentrated control framework will distribute the undertaking that Fig: 3Shows Resource Utilization goes to the framework by assessing the measure of assets

required in a conditional way and dependent on that its apportions the server with either high or low scoring. The most extreme the assets are required the server with most noteworthy score will be distributed and any place the low assets are required as server with less score will be relegated or allotted. With this a favorable position is the work or the errand that comes will be given to proper server and there by the undertaking finishes quicker and effectively. The server at that point will be prepared to 


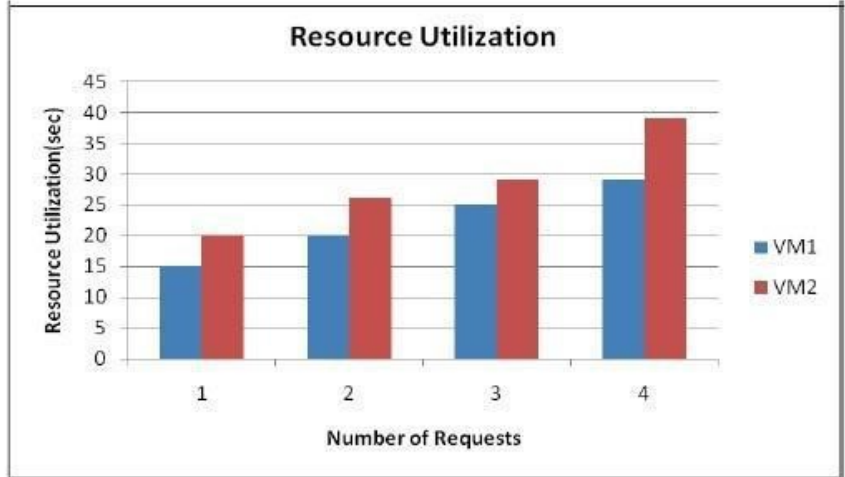

Fig:2 Represents the proposed load balancing.

All server will also have threshold level and this is also taken into account for allocation of task.

\section{Recreation toolboxs}

Considering unpredicted system condition and research facility asset scale (like servers), once in a while it is useful to and increasingly helpful for creating and running reenactment apparatuses to mimic huge scale tests. The exploration on powerful and enormous scale circulated condition can be satisfied by developing server farm recreation framework, which offers envisioned displaying and reenactment for huge scale applications in cloud foundation.

The server farm recreation framework can depict the application remaining task at hand articulation, which help us to know the measure of burden that should be doled out to that server, when the server arrives at that level the other server with next degree of score will be given the assignment.

incorporates client data, server farm position, the measure of clients and server farms, and the measure of assets in every datum center.10 Under the mimicked server farms, load adjusting calculations can be effectively actualized and assessed.

CloudSim: CloudSim is an occasion driven test system actualized in Java. As a result of its article arranged programming highlight, CloudSim permits augmentations and meaning of strategies in every one of the parts of the product stack, along these lines making it an appropriate research instrument that can emulate the complexities emerging from the environments. 11

CloudSched: CloudSched enables users to compare different resource scheduling algorithms in Infrastructure as a Service (IaaS) regarding both hosts and workloads. It can also help the developer identify and explore appropriate solutions considering different resource scheduling algorithms.

\section{CONCLUSION:}

Load Balancing is a necessary task in Cloud Computing environment to attain maximum use of resources. In this paper, we talk about Evaluation based load balancing method which helps in providing maximum efficiency in terms of execution and allocation of various tasks. The advantage of this is algorithm is most sought after resources task will get the maximum scored server to perform computation and least resource required task gets the least scored server. As high rated server is performing high rated task and low rated server is performing low rated task the resource utilization will happen in most appropriate manner.

\section{REFERENCES}

1. D. Saranya et.al, "Load Balancing Algorithms in Cloud Computing: A Review," International Journal of Advanced Research in Computer Science and Software Engineering, vol. 5, Issue 7, July 2015.

2. S. Sethi et.al, "Efficient Load Balancing in Cloud Computing using Fuzzy Logic," IOSR Journal of Engineering (IOSRJEN) ISSN: 2250 3021 vol. 2, pp. 65-71, July 2012.

3. T. Desai et.al, "A Survey of Various Load Balancing Techniques and Challenges in Cloud Computing," International Journal of Scientific \& Technology Research, vol. 2, Issue 11, November 2013.

4. S. Rajoriya et.al, "Load Balancing Techniques in Cloud Computing: An Overview," International Journal of Science and Researc h (IJSR), vol. 3, Issue 7, July 2014

5. Sharma S. et.al, "Performance Analysis of Load Balancing Algorithms," World Academy of Science, Engineering and Technology, 38, 2008.

6. Gross D. et.al, "Noncooperative load balancing in distributed systems", Elsevier, Journal of Parallel and Distributed Computing, No. 65, pp. 1022-1034, 2005

7. Nikravan M. et.al, "A Genetic Algorithm for Process Scheduling in Distributed Operating Systems Considering Load Balancing", Proceedings 21st European Conference on Modelling and Simulation (ECMS), 2007.

8. M. Amar et.al, "SLA Driven Load Balancing for Web Applications in Cloud Computing Environment",

9. Tian W, Xu M, Chen A, et al. Open-source simulators for cloud computing: Comparative study and challenging issues. Simul Modell Pract Theory. 2015;58:239-254.

10. Calheiros RN, Ranjan R, Beloglazov A, De Rose CA, Buyya R. Cloudsim: A toolkit for modeling and simulation of cloud computing environ-ments and evaluation of resource provisioning algorithms. Software: PractExper. 2011;41(1):23-50.

11. Tian W, Zhao Y, Xu M, Zhong Y, Sun X. A toolkit for modeling and simu-lation of real-time virtual machine allocation in a cloud data center. IEEE Trans AutomSci Eng. 2015;12(1):153-161. 